OPEN ACCESS

Edited by:

Gavin M. Bidelman,

The University of Memphis,

United States

Reviewed by:

Tobias Teichert,

University of Pittsburgh, United States

Claude Alain

Rotman Research Institute (RRI),

Canada

*Correspondence:

Kosuke Itoh

itoh@bri.niigata-u.ac.jp

${ }^{\dagger}$ Deceased

Specialty section:

This article was submitted to Auditory Cognitive Neuroscience,

a section of the journal

Frontiers in Neuroscience

Received: 12 February 2019

Accepted: 31 May 2019

Published: 24 June 2019

Citation:

Itoh K, Nejime M, Konoike N, Nakamura K and Nakada T (2019) Evolutionary Elongation of the Time

Window of Integration in Auditory

Cortex: Macaque vs. Human Comparison of the Effects of Sound

Duration on Auditory Evoked

Potentials. Front. Neurosci. 13:630.

doi: 10.3389/fnins.2019.00630

\section{Evolutionary Elongation of the Time Window of Integration in Auditory Cortex: Macaque vs. Human Comparison of the Effects of Sound Duration on Auditory Evoked Potentials}

\author{
Kosuke Itoh ${ }^{1 *}$, Masafumi Nejime ${ }^{2}$, Naho Konoike $^{2}$, Katsuki Nakamura ${ }^{2}$ and \\ Tsutomu Nakada'1+
}

${ }^{1}$ Center for Integrated Human Brain Science, Brain Research Institute, Niigata University, Niigata, Japan, ${ }^{2}$ Cognitive Neuroscience Section, Primate Research Institute, Kyoto University, Kyoto, Japan

The auditory cortex integrates auditory information over time to obtain neural representations of sound events, the time scale of which critically affects perception. This work investigated the species differences in the time scale of integration by comparing humans and monkeys regarding how their scalp-recorded cortical auditory evoked potentials (CAEPs) decrease in amplitude as stimulus duration is shortened from $100 \mathrm{~ms}$ (or longer) to $2 \mathrm{~ms}$. Cortical circuits tuned to processing sounds at short time scales would continue to produce large CAEPs to brief sounds whereas those tuned to longer time scales would produce diminished responses. Four peaks were identified in the CAEPs and labeled P1, N1, P2, and N2 in humans and mP1, mN1, mP2, and mN2 in monkeys. In humans, the N1 diminished in amplitude as sound duration was decreased, consistent with the previously described temporal integration window of $\mathrm{N1}$ (>50 ms). In macaques, by contrast, the $\mathrm{mN} 1$ was unaffected by sound duration, and it was clearly elicited by even the briefest sounds. Brief sounds also elicited significant $\mathrm{mN2}$ in the macaque, but not the human N2. Regarding earlier latencies, both P1 (humans) and $\mathrm{mP1}$ (macaques) were elicited at their full amplitudes even by the briefest sounds. These findings suggest an elongation of the time scale of late stages of human auditory cortical processing, as reflected by $\mathrm{N} 1 / \mathrm{mN} 1$ and later CAEP components. Longer time scales of integration would allow neural representations of complex auditory features that characterize speech and music.

Keywords: event-related potential, temporal integration, auditory late latency response, non-human primate, evolution

\section{INTRODUCTION}

Auditory information is integrated over time for obtaining neural representations of auditory events in the brain (Heil and Neubauer, 2001; Lerner et al., 2011; Farbood et al., 2015). The time scale of integration is a critical parameter of auditory processing that determines how sounds are represented in the brain and in perception (Moore, 2003; Sussman, 2005; Lerner et al., 2011, 2014; 
Farbood et al., 2015). For example, the perceptual distinction between voiced and unvoiced speech sounds (e.g., /ba/ vs. /pa/) depends on whether the voice onset interval is longer or shorter than about $30 \mathrm{~ms}$, respectively. A necessary condition for such perception to be possible is that the time scale for syllable perception is sufficiently longer than the voice onset interval.

Temporal integration occurs at each level of auditory processing, from peripheral to central, and the time scale of neural representations of continuously incoming sounds generally becomes longer at higher levels of sensory processing, reflecting the accumulation of information over time (Lerner et al., 2011; Hasson et al., 2015). Regarding the final stages of auditory processing that are directly relevant to the perception and cognition of sounds, the time scale of auditory cortical functions is implicitly assumed to be similar across human and non-human primate species. This assumption, however, has never been explicitly tested to our knowledge. It is worthwhile to examine the possibility that the time scale of auditory cortical processing has extended over the course of primate brain evolution to enable representations of complex sound features, such as those that characterize speech and music. In fact, the macaque homologs of human cortical auditory evoked potentials (CAEPs) have shorter latencies compared with the human CAEP (Fishman et al., 2000b; Itoh et al., 2015), which strongly suggests shorter time scales of auditory cortical processing in the macaque cortex.

This work investigates species differences in the time scale of auditory cortical processing by comparing how the $\mathrm{N} 1$ and other components of human and macaque CAEP diminish in amplitude as the sound duration is decreased from $100 \mathrm{~ms}$ or longer to $2 \mathrm{~ms}$. A long time scale of integration entails that CAEP amplitudes become small as sound duration is decreased, while neural circuits tuned to processing auditory signals at short times scales would continue to produce large CAEP responses to brief stimuli.

\section{MATERIALS AND METHODS}

\section{Subjects}

Animal experiments were carried out on four young adult rhesus monkeys (Macaca mulatta; two males and two females, $4-5 \mathrm{~kg}$, 4-7 years old). These animals displayed no behavioral signs of hearing deficits. The animals were not restricted from food and water throughout experimental period. The Animal Care and Use Committee of Kyoto University approved the study, and all experiments were performed in accordance with the Guide for the Care and Use of Laboratory Animals of the National Research Council (1996) and the Guide for Care and Use of Laboratory Primates of Kyoto University. All experiments were conducted at Kyoto University.

Human experiments were carried out on twelve, audiologically normal, right-handed volunteers (18-23 years old, two males). All volunteers provided written informed consent for participation prior to enrollment. Human experiments were conducted at the University of Niigata after the Internal Review Board of the University of Niigata approved the study. All experiments were performed in accordance with the Declaration of Helsinki.

\section{Stimuli and Apparatus}

The stimuli, apparatus, and procedure used were identical across the macaque and human experiments unless otherwise noted.

The auditory stimulus was a pure tone $(1500 \mathrm{~Hz})$ with variable durations of 200 (human experiment only), 100, 50, 10, 5, 3, or $2 \mathrm{~ms}$ with a linear rise/fall time of $1 \mathrm{~ms}$. The stimulus duration contained the rise and fall times. The use of the $200 \mathrm{~ms}$ stimulus was unnecessary in the macaque experiment, because all the macaque CAEP components occurred before $200 \mathrm{~ms}$ in latency, meaning that no further temporal integration would occur for this stimulus.

These sounds were presented in a randomized sequence with a variable interstimulus interval (ISI) of 300-400 ms. The distribution of the ISI within this interval was uniform. A total of 1400 trials in the monkey experiments and 600 in the human experiments were conducted for each variable duration condition. Sounds were digitally synthesized with Adobe Audition CS6, saved as wave files (16 bit, $48 \mathrm{kHz}$ ), and played back on a computer using Presentation software (Neurobehavioral systems, Berkeley, CA) and Sound Blaster audio hardware (Creative Technology, Jurong East, Singapore). Sound playback latencies with respect to the trigger signal were measured and corrected in post-processing. Sounds were presented using a loud speaker (MSP7 Studio; Yamaha, Hamamatsu, Japan) placed $0.6 \mathrm{~m}$ from the monkey's head, or $1.2 \mathrm{~m}$ from the human's head. The speaker was placed straight ahead of the subject for both species, and sound intensity was in the range of $65-70 \mathrm{~dB}$ SPL as measured at the position of the head.

In the animal experiments, the monkeys sat in a primate chair and passively listened to the sounds in a sound-attenuated room. Occasional rest phases were provided (approximately once every $20 \mathrm{~min}$ ) to check the state of the animal, provide food and/or water, and/or to maintain electrode impedance. Polystyrene blocks placed on the left and right sides of the monkey's head were used to restrict left-right rotational head movements. Horizontal bars placed above the nose and supraorbital ridge restricted upward rotations and forward movements. The detailed protocol of animal preparation has been described elsewhere (Itoh et al., 2015). In the human experiments, participants sat in a comfortable chair in a sound-attenuated room and listened passively to the sounds without being given specific instructions.

\section{CAEP Recording and Analyses (Macaques)}

Silver electrodes used for human sleep recordings (NE-136A, Nihon Kohden, Japan) were placed on the monkey's scalp and earlobes according to the International 10-20 system to record the electroencephalogram (EEG). An electrooculogram (EOG) was also recorded using an electrode placed to the lower left of the left eye to monitor ocular artifacts. After the electrodes were placed using collodion, electrode gel was applied to lower the impedance to below $5 \mathrm{k} \Omega$. Twelve electrodes were applied (F3, F4, C3, C4, P3, P4, Fz, Cz, Pz, 
A1, A2, and EOG). Because there was no clear or consistent left-right asymmetry, analyses were performed using only the midline electrodes $(\mathrm{Fz}, \mathrm{Cz}$, and $\mathrm{Pz})$ where the CAEP amplitudes were maximal. All EEG and EOG channels were referenced to $\mathrm{Cz}$ during the recordings and re-referenced to the linked earlobes (i.e., the average of A1 and A2) during post-processing. The EEG and EOG were amplified (16 bit, $0.1 \mu \mathrm{V} / \mathrm{LSB}$ precision), bandpass filtered $(0.016-250 \mathrm{~Hz})$, and sampled at $1000 \mathrm{~Hz}$ using BrainAmp MR plus (Brain Products, Munich, Germany).

In post-processing, the data were bandpass filtered (1$100 \mathrm{~Hz}, 12 \mathrm{~dB} / \mathrm{oct}$ ), segmented, time-locked to the onset of the stimulus ( -50 to $400 \mathrm{~ms}$ ), adjusted to the baseline using the prestimulus-period average, artifact filtered $( \pm 150 \mu \mathrm{V}$ relative to baseline), and then averaged to obtain the CAEPs. The number of non-rejected time segments was 1198-1231 (8688\%), 1194-1218 (85-87\%), 1312-1372 (94-98\%), and 13041317 (93-94\%), respectively, for the four monkeys. EMSE Suite version 5.5.1 (Source Signal Imaging, La Mesa, CA, United States) was used for the processing of electrophysiological data. The amplitude of $\mathrm{mP1}$ was represented by the average in the time range $20-40 \mathrm{~ms}$ at $\mathrm{Cz}$, while the time window for $\mathrm{mN} 1$ was $40-60 \mathrm{~ms}$ at $\mathrm{Cz}$ and that for $\mathrm{mN} 2$ was 90-110 $\mathrm{ms}$ at $\mathrm{Fz}$ or $\mathrm{Cz}$. These time slots and electrodes were determined by examining the group-averaged CAEP waveforms (Figure 1). For each component, the time window was centered at the peak latency, and the electrode of analysis represented the spatial peak of the response in the 2 ms condition.

\section{CAEP Recording and Analyses (Humans)}

Electroencephalograms were recorded from five silver electrodes placed along the midline ( $\mathrm{Fpz}, \mathrm{Fz}, \mathrm{Cz}, \mathrm{Pz}$, and $\mathrm{Oz}$ ) and the left and right earlobes (A1 and A2) according to the International 10-20 system. Horizontal and vertical EOGs (HEOG and VEOG) were recorded from the left eye. The EEG and EOG were amplified (16 bit, $0.1 \mu \mathrm{V} / \mathrm{LSB}$ precision), bandpass filtered $(0.016-250 \mathrm{~Hz})$, and sampled at $1000 \mathrm{~Hz}$ using BrainAmp MR plus (Brain Products, Munich, Germany). The data were re-referenced offline to the linked ears.

The procedures for obtaining the human CAEPs were identical to those used in the animal experiments, except that the criteria for artifact rejection was $\pm 100 \mu \mathrm{V}$. The number of non-rejected epochs was in the range of 351-571 (59-95\%) with a mean of $508(85 \%)$. The time windows and electrodes chosen to evaluate the CAEP amplitudes were 45-65 ms at Cz, respectively, for $\mathrm{P} 1$. Because the peak latency of $\mathrm{N} 1$ changed noticeably with sound duration, the time window for the N1 analyses was $80-110 \mathrm{~ms}$ for the $50 \mathrm{~ms}$ and $100 \mathrm{~ms}$ conditions and 105-135 ms for the other conditions, all at the Fz electrode. The amplitude of P1-N1 deflection was also calculated, by subtracting the P1 amplitude at Fz from the N1 amplitude at Fz. For the N2 component, the time window was $150-250 \mathrm{~ms}$ at Fz. These time slots and electrodes were determined by examining the groupaveraged CAEP waveforms (Figure 1). For each component, the time window was centered at the peak latency, and the electrode represented the spatial peak of the response in the $2 \mathrm{~ms}$ condition.

\section{Species Comparisons}

We analyzed and interpreted our results per the hypothesis that the human P1-N1-P2- N2 complex corresponds, peak-topeak, to the macaque mP1-mN1-mP2-mN2 complex (Fishman et al., 2000b, 2014; Fishman and Steinschneider, 2012; Itoh et al., 2015). Rationales for this assumption are presented in the Discussion section.

Considering the latency of $\mathrm{N} 1$ and $\mathrm{mN1}$, which were approximately $100 \mathrm{~ms}$ or shorter, the amplitudes of these waves in the $100 \mathrm{~ms}$ condition represented their (nearly) maximum amplitude (for the present ISI) because further temporal integration could not contribute to an amplitude increase. For the same reason, the $\mathrm{P} 1 / \mathrm{mP} 1$ amplitudes in the 50 and $100 \mathrm{~ms}$ conditions, the N2 amplitude in the $200 \mathrm{~ms}$ condition, and the $\mathrm{mN} 2$ amplitude in the $100 \mathrm{~ms}$ condition were also representative of their maximum amplitudes These situations allowed us to quantitatively analyze how $\mathrm{P} 1 / \mathrm{mP} 1, \mathrm{~N} 1 / \mathrm{mN} 1$, and $\mathrm{N} 2 / \mathrm{mN} 2$ diminished in amplitude from their maximum values as sound duration decreased.

Species differences for the effects of stimulus duration on $\mathrm{P} 1 / \mathrm{mP} 1, \mathrm{~N} 1 / \mathrm{mN} 1$, and $\mathrm{N} 2 / \mathrm{mN} 2$ were statistically evaluated using a mixed linear model using a random intercept and slope for each subject and a variance component covariance structure and maximum likelihood estimation. Each data sample in the analysis represented one epoch of non-rejected EEG recording data. Species (Human/Macaque) was a betweensubject factor, and Duration (2/3/5/10/50/100 $\mathrm{ms}$ in the Macaque, and $2 / 3 / 5 / 10 / 50 / 100 / 200 \mathrm{~ms}$ in the Human) was a withinsubject factor. That is, the regression equation included the design matrix that modeled the fixed effects of Species, Duration, and their interaction, as well as the design matrix for the random effects of Duration that modeled random intercepts and slopes at the subject level. Pairwise comparisons between the stimuli conditions were performed in each species to reveal the pattern of amplitude change across different stimulus durations. These results were adjusted for multiple comparisons by using the method of Šidák (1967). Statistical analyses were performed using IBM SPSS Statistics version 22 (IBM, Armonk, NY, United States).

\section{RESULTS}

\section{Overview}

Grand-averaged waveforms of the human and macaque CAEPs to pure-tone stimuli of different durations are shown in Figure 1. As a general finding, the human and macaque CAEPs had the same number of peaks with matched polarities, although the latencies were overall shorter in the macaque. The P1 $(55 \mathrm{~ms}$, peak latency), N1 (90-130 ms), P2 (160 ms), and N2 (200 ms) waves constituted the human CAEP. The peak latencies of P1 and $\mathrm{N} 1$ were longer for brief ( $\leq 10 \mathrm{~ms}$ ) sounds than for long sounds. The N2 response was weak and difficult to identify in most conditions. The macaque CAEP also comprised a series of four transient responses, which were labeled as macaque $\mathrm{P} 1$ ( $\mathrm{mP} 1$, $30 \mathrm{~ms}$ ), macaque $\mathrm{N} 1$ ( $\mathrm{mN} 1,55 \mathrm{~ms})$, macaque P2 (mP2, $70 \mathrm{~ms}$ ) and macaque $\mathrm{N} 2(\mathrm{mN} 2,95 \mathrm{~ms})$, to indicate their polarity and 
Human

$\mathrm{Fz}$

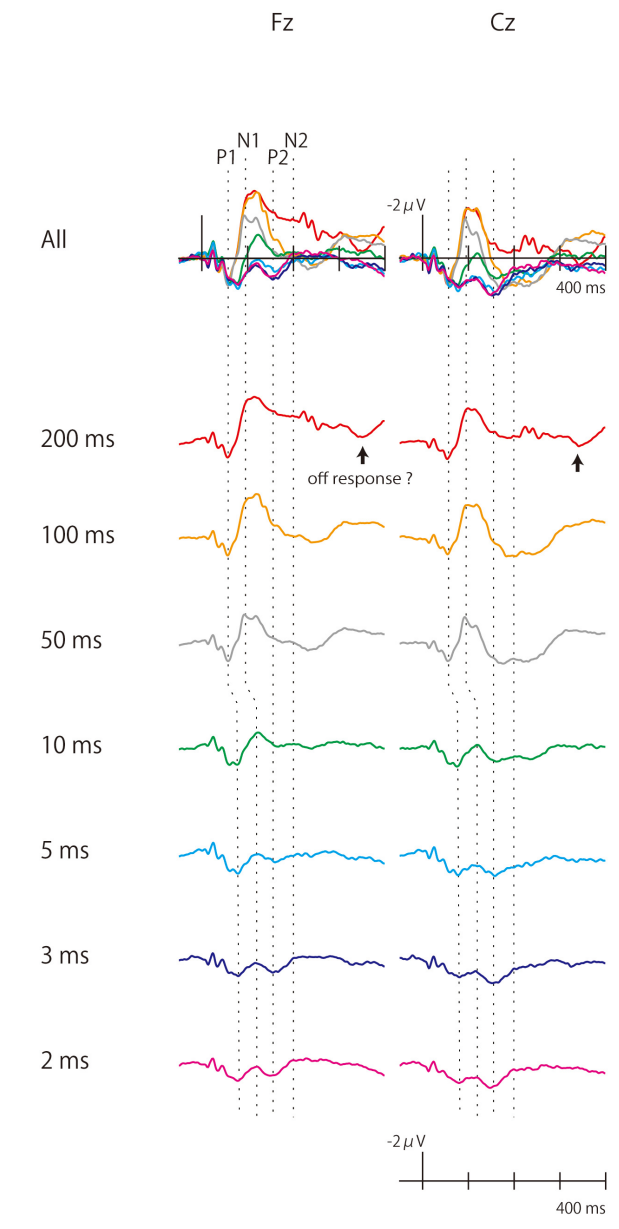

Macaque

$\mathrm{Fz}$

Cz

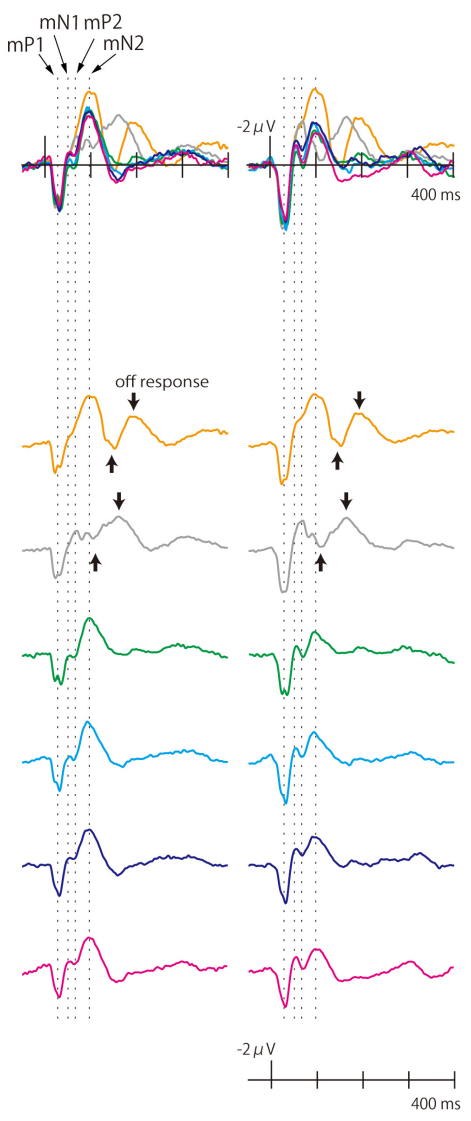

FIGURE 1 | Human and monkey scalp-recorded CAEPs elicited by pure-tone stimuli of different durations. The macaque cortex continued to produce large CAEPs as sound duration was shortened. Off-responses were observed more clearly in monkeys.

order (Itoh et al., 2015). In addition, off responses, or positivenegative deflections that were time-locked to sound offset, were unambiguously elicited by the $50 \mathrm{~ms}$ and $100 \mathrm{~ms}$ stimuli in monkeys, while such responses were not clearly identified in the human CAEP (Figure 1).

Sound duration affected the CAEP waveforms in both humans and macaques, but in different manners (Figure 1). In humans, the auditory cortical responses diminished in amplitude as sound duration was shortened, and waves later than P1 were not clearly elicited by stimuli shorter than $10 \mathrm{~ms}$. In monkeys, by contrast, substantial cortical responses remained even to the briefest sound, and the full complex of mP1-mN1-mP2-mN2 peaks was clearly identified in the $2 \mathrm{~ms}$ condition. These observations were supported statistically as described below.

\section{Effects of Sound Duration $\mathrm{P} 1$ and $\mathrm{mP1}$}

In both humans and macaques, a sound duration of as short as $2 \mathrm{~ms}$ was sufficient to elicit significant $\mathrm{P} 1 / \mathrm{mP} 1$ responses
(Figure 1). The amplitudes of $\mathrm{P} 1$ and $\mathrm{mP1}$ were significantly different from the baseline of zero microvolt in all stimulus conditions, including the $2 \mathrm{~ms}$ condition (Table 1). Furthermore, the P1 and $\mathrm{mP1}$ amplitudes did not increase with the duration of the stimulus (Figure 2). The mixed linear model analysis revealed that the effect of Duration was not significant, $F(6,91.0)=1.2$, $p=0.311$, and the Species $\times$ Duration interaction was significant, $F(5,82.0)=2.4, p=0.048$. When analyzed separately in each species, the effect of Duration was not significant in humans, $F(6,69.8)=1.7, p=0.136$, nor in monkeys, $F(5,19.7)=1.3$, $p=0.294$. These results indicated that the time window of integration was quite short $(<2 \mathrm{~ms})$ for P1 and $\mathrm{mP} 1$.

For a more direct species comparison of the effects of sound duration on the $\mathrm{P} 1$ and $\mathrm{mP} 1$ as well as other CAEP components, group-averaged CAEP amplitudes were obtained for each species after the amplitudes were normalized to $z$-scores across the stimulus conditions for each subject (Figure 3). The results confirmed the small effects of sound duration on the mP1 and P1 amplitudes. Although the human P1 amplitude appeared to 
decrease slightly with sound duration, this could be explained by an overlap of the rising phase of $\mathrm{N} 1$ that significantly increased in amplitude with sound duration (see the next section).

\section{$\mathrm{N} 1$ and $\mathrm{mN} 1$}

We observed a significant species difference in the effects of sound duration on the $\mathrm{mN1}$ and $\mathrm{N} 1$ amplitudes (Figures 2, 3). The Species $\times$ Duration interaction was significant, $F(5,67.4)=6.8, p<0.001$, and, when tested separately in each group, the effect of Duration was significant in humans, $F(6,71.6)=27.2, p<0.001$, but not in monkeys, $F(5,19.8)=1.3$, $p=0.306$. In humans, pairwise comparisons indicated that the $\mathrm{N} 1$ amplitudes in the 2, 3, 5, and $10 \mathrm{~ms}$ conditions were all significantly smaller compared to $100 \mathrm{~ms}$ or longer stimuli, $p<0.05$ (Šidák -corrected) (Table 1). In monkeys, none of the pairwise comparisons were significant, $p>0.05$ (Šidákcorrected) (Table 1).

\section{$\mathrm{N} 2$ and Frontal mN2}

The analysis of $\mathrm{mN} 2$ and $\mathrm{N} 2$ amplitudes was made difficult by the fact that a substantial off-response overlapped the macaque $\mathrm{mN} 2$ when the sound duration was $50 \mathrm{~ms}$ (Figure 1). The sound offset evoked a positive wave at a latency of $50 \mathrm{~ms}$ (Figure 1), resulting in an apparent decrease in the amplitude of the onsetevoked $\mathrm{mN} 2$ when the sound duration was $50 \mathrm{~ms}$ (Figures 1-3). Therefore, the results were interpreted with caution regarding the $50 \mathrm{~ms}$ condition in monkeys.

We first analyzed the $\mathrm{mN} 2$ measured at the Fz electrode, referred to here as the frontal $\mathrm{mN} 2$. The rationale for this choice of electrode was due to the greatest $\mathrm{mN} 2$ amplitude measured at $\mathrm{Fz}$, except for the $100 \mathrm{~ms}$ condition (Figure $\mathbf{1}$ and Table $\mathbf{1}$ ).

The regression analysis revealed that the Species $\times$ Duration interaction was significant, $F(5,84.5)=10.0, p<0.001$, indicating that the frontal $\mathrm{mN} 2$ and $\mathrm{N} 2$ were affected differently by sound duration. When tested separately in each species, the effect of Duration was significant in humans, $F(6,69.7)=22.5, p<0.001$, and pairwise comparisons revealed that the $\mathrm{N} 2$ amplitude was greater for the $200 \mathrm{~ms}$ stimulus compared to all other conditions, $p<0.05$ (Šidák-corrected) (Table 1). The effect of Duration was also significant in monkeys, $F(5,19.4)=11.7, p<0.001$. In the post-hoc analysis, all pairwise comparisons with the $50 \mathrm{~ms}$ condition were significant, which were explained by the overlap of the off-response. More importantly, there was a significant difference in the frontal $\mathrm{mN} 2$ amplitude between the $100 \mathrm{~ms}$ and the $2 \mathrm{~ms}$ conditions, $p<0.05$ (Šidák-corrected), but not in any other pairwise comparisons (Table 1).

Similar results were obtained when the entire analysis was performed after excluding the data for the $50 \mathrm{~ms}$ condition in monkeys. The Species $\times$ Duration interaction was significant, $F(4,74.9)=2.8, p=0.030$, and the effect of Duration was significant in humans (as shown above) and in monkeys, $F(4,15.2)=4.2, p=0.017$. In the macaque, pairwise comparisons again revealed a significant difference in the frontal $\mathrm{mN} 2$ amplitude between the $100 \mathrm{~ms}$ and $2 \mathrm{~ms}$ conditions, $p<0.05$ (Šidák-corrected), but not in any other pairwise comparisons.

Importantly, the frontal $\mathrm{mN} 2$ amplitude was significantly different from the baseline of zero microvolt in all conditions except the $50 \mathrm{~ms}$ condition, as determined by the $95 \%$ confidence interval (Table 1): this was true even for the briefest stimulus. Individual monkeys' response profiles confirm significant frontal $\mathrm{mN} 2$ responses to brief sounds (Figure 2), and the CAEP waveforms also show clear frontal $\mathrm{mN} 2$ responses to these stimuli (Figure 1). These results indicated that a few $\mathrm{ms}$ of temporal integration was sufficient to elicit a significant frontal $\mathrm{mN} 2$ in monkeys.

In humans, by contrast, the $\mathrm{N} 2$ amplitude significantly diminished as the sound duration was shortened from 200 to $100 \mathrm{~ms}$ (Figures 1, 2), and the amplitude was not significantly different from the basline in all conditions except the $200 \mathrm{~ms}$ condition, as determined by the $95 \%$ confidence interval of the estimates of the N2 amplitude (Table 1). These results indicated that the time window of integration for the N2 was longer than $100 \mathrm{~ms}$ in humans.

\section{Central mN2}

The waveforms in Figure 1 indicate a large mN2-like response elicited by the $100 \mathrm{~ms}$ stimulus at the $\mathrm{Cz}$ electrode, although the $\mathrm{mN} 2$ amplitude was always greatest at $\mathrm{Fz}$ in all other conditions. In general, a difference in scalp distribution indicates different neural sources. Therefore, whether the $\mathrm{Cz}$ maximal mN2-like response in the $100 \mathrm{~ms}$ condition was generated by the same neural sources as those that generated

TABLE 1 | CAEP amplitudes.

\begin{tabular}{|c|c|c|c|c|c|c|c|}
\hline Duration (ms) & \multicolumn{3}{|c|}{ Humans } & \multicolumn{4}{|c|}{ Macaques } \\
\hline 2 & $1.3^{\dagger}[0.8,1.9]$ & $0.4^{*}[-0.6,1.3]$ & $0.1^{*}[-0.4,0.6]$ & $2.8^{\dagger}[1.8,3,8]$ & $-0.7[-2.1,0.7]$ & $-3.1^{* \dagger}[-5.8,-0.4]$ & $-2.0^{*}[-5.6,1.6]$ \\
\hline 3 & $1.2^{\dagger}[0.7,1.8]$ & $0.4^{*}[-0.5,1.4]$ & $0.3^{*}[-0.2,0.8]$ & $2.9^{\dagger}[1.9,3.9]$ & $-1.0[-2.4,0.4]$ & $-3.3^{\dagger}[-6.0,-0.6]$ & $-2.6^{*}[-6.3,0.9]$ \\
\hline 5 & $1.4^{\dagger}[0.8,2.0]$ & $0.2^{*}[-0.8,1.1]$ & $0.3^{*}[-0.2,0.8]$ & $3.4^{\dagger}[2.5,4.4]$ & $-0.5[-1.9,0.9]$ & $-3.4^{\dagger}[-6.1,-0.7]$ & $-2.4^{*}[-6.0,1.2]$ \\
\hline 10 & $1.1^{\dagger}[0.5,1.6]$ & $-0.9 *[-1.8,0.1]$ & $-0.2^{*}[-0.7,0.3]$ & $3.6^{\dagger}[2.6,4.6]$ & $-0.0[-1.4,1.3]$ & $-3.4^{\dagger}[-6.2,-0.7]$ & $-2.0^{*}[-5.6,1.6]$ \\
\hline 200 & $1.0^{\dagger}[0.4,1.5]$ & $-2.3^{\dagger}[-3.3,-1.4]$ & $-1.8^{\dagger}[-2.3,-1.3]$ & N.A. & N.A. & N.A. & N.A. \\
\hline
\end{tabular}

The values indicate the mean and the $95 \%$ confidence interval in $\mu \mathrm{V}$. Asterisks $\left(^{*}\right)$ denote that the amplitude was significantly smaller compared to the $100 \mathrm{~ms}$ condition or the 200 ms condition, $P<0.05$ (Šidák-corrected). Daggers ( $\left(^{\dagger}\right)$ denote that the amplitude was significantly different from the baseline as determined by the $95 \%$ confidence interval. N.A.: data not available. 


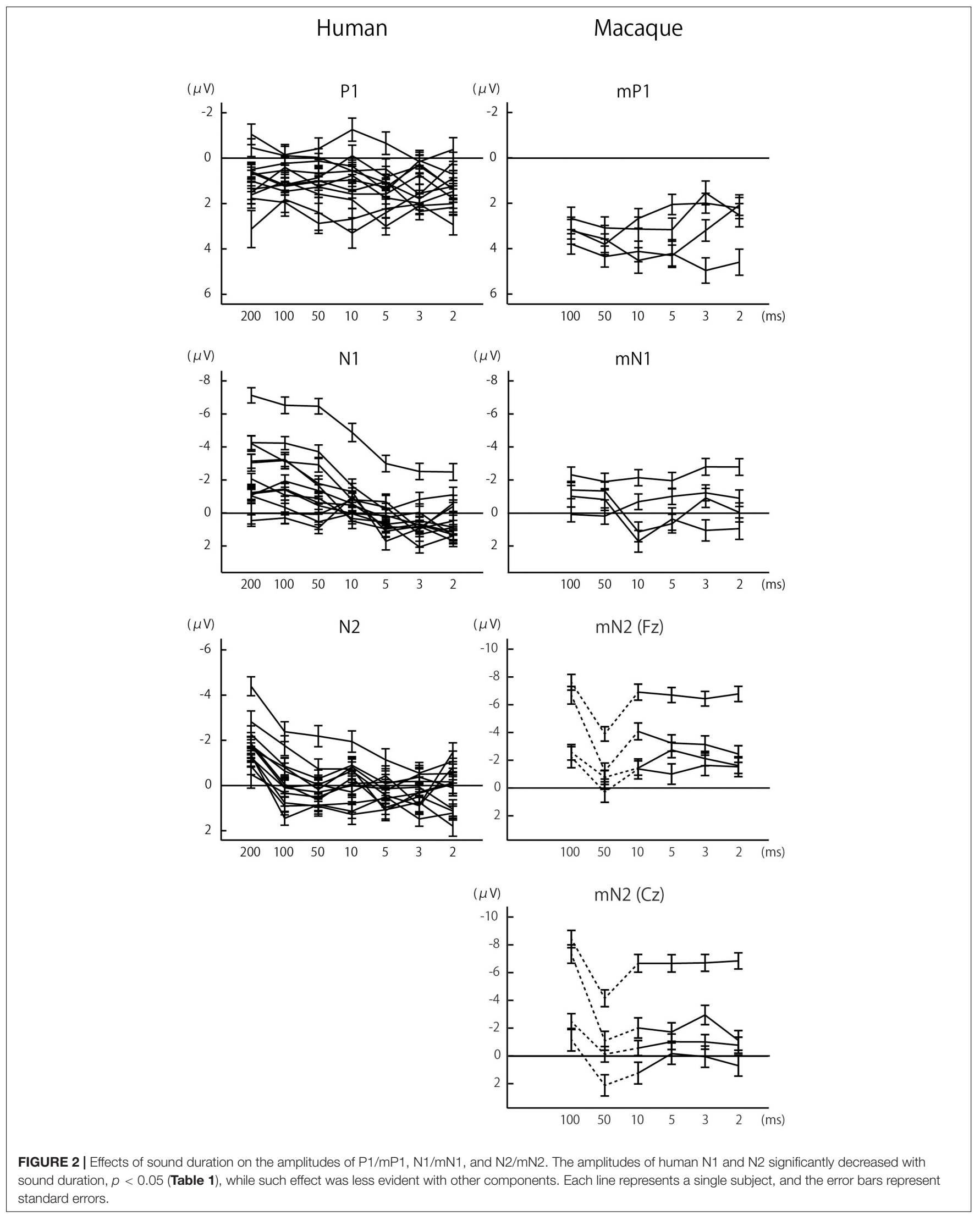




\section{$\mathrm{P} 1 / \mathrm{mP} 1$}

$\mathrm{N} 1 / \mathrm{mN} 1$

$\mathrm{N} 2 / \mathrm{mN} 2$

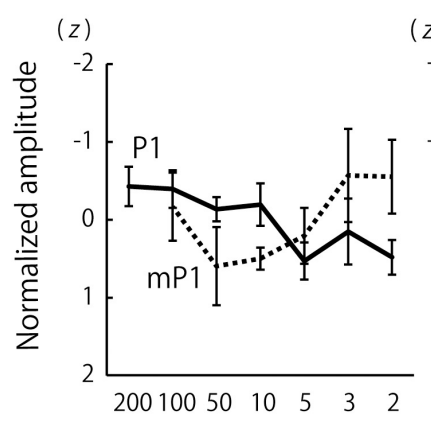

( $z$ )

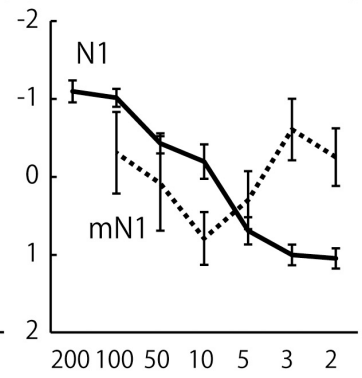

(z)

Sound duration

FIGURE 3 | Group comparisons of the effects of sound duration on the amplitudes of P1/mP1, N1/mN1, and N2/mN2. The lines represent group-averages that were calculated after the CAEP amplitudes were normalized to z-scores across the sound duration conditions for each subject. The error bars represent standard errors.

the Fz-maximal $\mathrm{mN} 2$ response in other stimulus conditions was questionable.

One possible explanation was that, as the stimulus duration increased, a novel CAEP component with a central distribution began to overlap the genuine $\mathrm{mN} 2$ that had a frontal distribution. Another equally valid interpretation was to consider $\mathrm{mN} 2$ as a composite wave that had multiple generators: one frontal subcomponent maximal at $\mathrm{Fz}$ and another central subcomponent at $\mathrm{Cz}$. This is a matter of definition, and we have decided to take this latter view.

To examine how the central subcomponent of $\mathrm{mN} 2$, or central $\mathrm{mN} 2$, was affected by sound duration, the statistical analyses in section $\mathrm{N} 2$ and Frontal $\mathrm{mN} 2$ were repeated using the $\mathrm{Cz}$ electrode. The regression analysis revealed that the Species $\times$ Duration interaction was significant, $F(5,88.2)=12.2$, $p<0.001$, indicating that the central $\mathrm{mN} 2$ and $\mathrm{N} 2$ were affected differently by sound duration. When tested separately in each species, the effect of Duration was significant in humans (see Section 3.2.3) and in monkeys, $F(5,19.3)=13.3, p<0.001$. In the post-hoc analysis of central $\mathrm{mN} 2$, all pairwise comparisons with the $100 \mathrm{~ms}$ condition were significant, $p<0.05$ (Šidákcorrected) (Table 1).

Similar results were obtained when the entire analysis was performed after excluding the data for the $50 \mathrm{~ms}$ condition in monkeys. The Species $\times$ Duration interaction was significant, $F(4,74.9)=9.4, p<0.001$, and the effect of Duration was significant in humans (as shown above) and in monkeys, $F(4,15.2)=8.9, p<0.001$. In the macaque, pairwise comparisons revealed a significant difference in the central $\mathrm{mN} 2$ amplitude between the $100 \mathrm{~ms}$ and all other conditions, $p<0.05$ (Šidák-corrected).

These results suggested that the integration window for the central $\mathrm{mN} 2$ was between 50 and $100 \mathrm{~ms}$, whereas that for the human N2 was between 100 and $200 \mathrm{~ms}$. In contrast to the frontal $\mathrm{mN} 2$, which was elicited by the briefest stimulus, the central $\mathrm{mN} 2$ amplitude at $\mathrm{Cz}$ was not significantly different from zero for sounds shorter than $100 \mathrm{~ms}$, as determined by the $95 \%$ confidence interval (Table 1).

\section{DISCUSSION}

Temporal integration is a fundamental principle of neural processing, and the time scale of integration is one of the most important parameters of brain function. Analogous to how a large receptive field in vision allows neural representations of complex figures that extend over space, a long time scale in auditory processing enables representations of complex auditory features due to accumulation of information over time. This work provides the first experimental evidence, to our knowledge, that the human brain has a longer time scale than the macaque brain regarding late stages of auditory cortical processing as indexed by N1 and later components of CAEP.

The human N1 significantly decreased in amplitude as sound duration was shortened from $100 \mathrm{~ms}$ to $2 \mathrm{~ms}$. This is consistent with the previously described temporal integration window of $\mathrm{N1}$, which is $50 \mathrm{~ms}$ or longer (Onishi and Davis, 1968; Alain et al., 1997). While the temporal integration window of macaque $\mathrm{mN} 1$ is unknown, its latency ( $55 \mathrm{~ms}$ ) constrains it to be shorter than the temporal integration window of human N1. Our experiment provided empirical proof of this notion by demonstrating that substantial $\mathrm{mN} 1$ responses persist for brief stimuli that are too short to elicit a clear N1 in humans.

Further evidence for a shorter time scale of auditory processing in monkeys was obtained by CAEP components elicited after $\mathrm{N} 1$ in latency. A clear frontal $\mathrm{mN} 2$ response was elicited by very brief sounds $(<10 \mathrm{~ms})$ at the $\mathrm{Fz}$ electrode in monkeys, indicating a short time window of temporal integration for the frontal subcomponent of $\mathrm{mN} 2$. The central subcomponent of $\mathrm{mN} 2$ measured at $\mathrm{Cz}$ had a longer time window of integration (50-100 ms), but it was shorter than that of the human N2 (>100 ms). These contrasting results regarding the frontal and the central subcomponents of $\mathrm{mN} 2$ could be interpreted in two ways. First, it was possible that a common set of neurons that generated both the frontal $\mathrm{mN} 2$ and central $\mathrm{mN} 2$ had a relatively long time window of integration (>50 ms), but that it was also activated by very short stimuli; that is, there were two stages of temporal integration, one occurring early, 
and another occurring late. Second, it was also possible that the frontal $\mathrm{mN} 2$ and the central $\mathrm{mN} 2$ reflected the neural activities of different ensembles of neurons, as suggested by the difference in their scalp distributions. In this case, it would be reasonable to conclude that the frontal $\mathrm{mN} 2$ and the central $\mathrm{mN} 2$ had different time windows of integration, albeit the similarity in peak latency. Although there was an abrupt increase in the mN2 amplitude at $\mathrm{Fz}$ between the $50 \mathrm{~ms}$ and $100 \mathrm{~ms}$ conditions, this could be interpreted as an overlap of the central $\mathrm{mN} 2$ rather than an increase in the frontal $\mathrm{mN} 2$ amplitude itself.

In addition, robust off-responses were elicited by long stimuli (50 and $100 \mathrm{~ms}$ ) in monkeys, while such waves were difficult to identify in humans. The latter result is consistent with previous findings that off-responses in humans are typically elicited with sounds longer than $100 \mathrm{~ms}$ (Hillyard and Picton, 1978; Pantev et al., 1996). To the best our knowledge, this is the first report to describe scalp-recorded auditory off-responses in the macaque monkey.

We do not assert that the absence of $\mathrm{N} 1 / \mathrm{mN} 1$ and later CAEP responses to brief sounds signified that the brief stimuli were undetected in the auditory cortex. These sounds, in fact, evoked clear mP1/P1 in both humans and monkeys. Because the temporal integration windows of $\mathrm{P} 1$ and $\mathrm{mP} 1$ must be shorter than their latencies (50 and $30 \mathrm{~ms}$, respectively), their amplitudes in the 50 and $100 \mathrm{~ms}$ conditions represented their saturated maxima. As shortening the sound duration to $2 \mathrm{~ms}$ did not result in any significant decrease in amplitude from these maxima, the time scale of auditory processing at an initial stage of cortical processing as indexed by $\mathrm{P} 1 / \mathrm{mP} 1$ was quite short $(<2 \mathrm{~ms})$, both in humans and monkeys. Our P1 finding in the human participants confirm, and also extends, an earlier magnetoencephalographic observation that the magnetic counterpart of P1 (or P1m) was barely affected by the duration of sound in the range of 34-76 ms (Ross et al., 2009).

Importantly, the above discussions are based on the premise that the $\mathrm{N} 1$ and $\mathrm{mN} 1$ represent functional homologs of each other. Establishing a cross-species correspondence of evoked potential components is a difficult issue. However, two lines of evidence support the assumption of N1-mN1 correspondence: morphological and functional. First, there is a one-to-one correspondence in the morphological structure of human and macaque CAEPs, when they are recorded over the scalp. Both the human and macaque scalp-recorded CAEP comprise four transient responses followed by a sustained potential, whose polarities are completely matched (Itoh et al., 2015), and the simplest interpretation would be that the human P1-N1-P2$\mathrm{N} 2-\mathrm{SP}$ peaks correspond to mP1-mN1-mP2-mN2-mSP peaks in the macaque, respectively. The latencies of the peaks are overall shorter in the macaque, but it is a general property of the macaque event-related potentials (Woodman, 2012). Considering that the macaque auditory evoked responses already have shorter latencies at the level of brainstem (Legatt et al., 1986), the fact that the CAEP latencies are short in the macaque in fact supports, rather than argues against, the proposed correspondence.

Second, and more important, $\mathrm{mN1}$ represents a stage of auditory processing that is functionally comparable to that represented by the human $\mathrm{N} 1$. In humans, a contextually "deviant" stimulus elicits a mismatch negativity (MMN), reflecting preattentive detection of a change in acoustic pattern established by a repetitive stimulus train (Naatanen et al., 2007). MMN begins around the latency of N1 (100 ms) and peaks at 150-250 ms post-stimulus, indicating that the preattentive change detection mechanism operates at the level of auditory processing as indexed by N1-P2. On the other hand, the macaque counterpart of $\mathrm{MMN}$ has a peak latency of 80-90 ms (Javitt et al., 2000; Honing et al., 2012; Gil-da-Costa et al., 2013), commencing around mN1 (50 $\mathrm{ms}$ ) and overlapping $\mathrm{mP} 2(70 \mathrm{~ms})$, which is clearly earlier than $\mathrm{mN2}$. Thus, it is reasonable to assume that $\mathrm{N} 1$ and $\mathrm{mN} 1$ reflect functionally comparable stages of auditory processing. This argument is further corroborated by an eventrelated potential component called object-related negativity (ORN), which reflects segregation of concurrent auditory objects, as it overlaps N1-P2 in humans (Alain et al., 2002; Alain and McDonald, 2007) and mN1-mP2 in monkeys (Fishman et al., 2014).

A competing hypothesis is that the frontal $\mathrm{mN} 2$ or the central $\mathrm{mN} 2$, not $\mathrm{mN} 1$, represents the human homolog of N1. A rationale for this proposition is that they are all prominent vertex negativities at approximately $100 \mathrm{~ms}$. However, this tenet has several limitations. First it does not take into account the accumulating observation that evoked responses in the macaque generally have shorter latencies than those in humans (Legatt et al., 1986; Woodman, 2012). Second, it is also incompatible with the fact that MMN and ORN in macaque are elicited before the two subcomponents of mN2. Third, intracranial recordings of AEP from the supragranular layer of macaque primary auditory cortex have identified a negative peak around 50-60 ms (Fishman et al., 2000a, 2014; Fishman and Steinschneider, 2012), which likely contributes to our scalprecorded $\mathrm{mN} 1$.

However, setting putative homologies aside, it is important to note that the central $\mathrm{mN} 2$ has the same latency, polarity and time window of integration as the N1. This highlights the fact that there is clear evidence for extended temporal integration in the macaque that matches that observed in the human N1, albeit not in the presumed functional homolog of the $\mathrm{N} 1$, the $\mathrm{mN} 1$, but rather the central $\mathrm{mN} 2$ which emerges at a similar latency as the N1. Similar latencies may be more important than homology in this specific context, given that latency imposes a strict upper limit on the time window of integration of a component. However, it is also important to point out that the frontal $\mathrm{mN} 2$ which occurs at the same latency as the N1, seems to have a very short integration window. This presumed co-existence of short and long temporal integration windows for components at similarly long latencies establishes an overall different pattern of temporal integration between the two species.

In contrast to the present study, previous invasive recordings of macaque auditory cortical responses to sounds have identified a negative peak in the latency range of $70-80 \mathrm{~ms}$ as a putative homolog of the human $\mathrm{N} 1$; this macaque component has been labeled as N70 (Arezzo et al., 1975), N1 (Javitt et al., 2000), or 
N85 (Teichert, 2016). There are several possible reasons for the discrepancy. First, the N70 (Arezzo et al., 1975) was recorded using epidural electrodes placed on the lateral surface of the frontal lobe, which is consistent with a radially oriented source in the frontal cortex. On the other hand, our use of midline electrodes was suitable for recording neural activities that are generated by tangentially oriented sources on the superior plane of the temporal lobe, such as the human vertex N1. In fact, the same intracranial study (Arezzo et al., 1975) recorded two negative responses, N60 and N100, when depth electrodes were placed in the superior temporal gyrus. It is therefore possible that the $\mathrm{N} 60$ and $\mathrm{N} 100$ correspond to the $\mathrm{mN} 1$ and $\mathrm{mN} 2$, respectively. A problem with this explanation, however, is that (Arezzo et al., 1975) did not report these negativities as being evident at epidural electrodes that were placed close to the midline. In addition, the N1 (Javitt et al., 2000) and N85 (Teichert, 2016) were recorded using midline electrodes. Second, the macaque N1 (Javitt et al., 2000) was defined as the most negative peak in the time window of 40-120 ms, and this wide latency range would have included both the $\mathrm{mN} 1$ and $\mathrm{mN} 2$ without distinguishing them. Third, whereas all of the above studies, including ours, used electrodes placed at the earlobe or mastoid as the reference, the N85 (Teichert, 2016) was recorded using a reference placed at $\mathrm{Oz}$. It is difficult to compare wave morphologies if different references were used. Nevertheless, the waveforms in (Javitt et al., 2000) also show a negative peak at $\mathrm{Cz}$ around $80 \mathrm{~ms}$, which is similar in scalp distribution and latency to the N85 (Teichert, 2016).

The effect of sound duration on mN2 amplitude was different between the $\mathrm{Fz}$ and $\mathrm{Cz}$ electrodes. We took this finding to indicate that the $\mathrm{mN} 2$ had multiple generators which had different time windows of integration. The frontal subcomponent maximal at Fz was elicited by even the briefest sound, indicating a quite short time window. By contrast, the temporal integration window for the central $\mathrm{mN} 2$ was much longer, because it was estimated to be between 50 and $100 \mathrm{~ms}$. Importantly, both of these time windows were shorter than the time window for the human N2, which was clearly longer than $100 \mathrm{~ms}$.

There were several limitations in this study. First, although the sample size of four was large compared with the sample size of many macaque experiments, the number of subjects was nevertheless smaller in the animal study compared with the human study. This led to an unbalance in the statistical power for detecting effects in the post-hoc analyses (Table 1). Second, the lack of a CAEP amplitude modulation with sound duration is not necessarily evidence for an absence of temporal integration. It is possible that the macaque cortex integrates auditory information by mechanisms that are not captured by this effect. Third, and most critical, our analyses and interpretations of the CAEP findings are based on the putative homologies between the macaque and human CAEP components, which remains to be proven by future research. An alternative interpretation, which focuses on the absolute latency of the neural responses rather than on the homologies of the components, is that the auditory cortices of humans and macaques have a comparable time window of integration, as the negative peak at around $100 \mathrm{~ms}$ (i.e., the N1 in humans and the
$\mathrm{mN} 2$ in macaques) was modulated similarly by sound duration in both species.

\section{CONCLUSION}

In conclusion, this study provides novel evidence for a species difference in the time scale of auditory processing at late stages of auditory cortical processing as indexed by $\mathrm{N} 1$ and later components of CAEP. The time scale of auditory cortical processing affects many, if not all, aspects of auditory perception, such as loudness (Fastl and Zwicker, 2007), speech (Lerner et al., 2011, 2014), and music (Lalitte and Bigand, 2006; Farbood et al., 2015) perception. To speculate, the shorter time window of auditory integration in monkeys might be related to the limited complexity and flexibility of their vocalizations. The repertoire of non-human primate vocalization is limited compared to the human speech (Gustison et al., 2012), and it is difficult to train non-human primates to produce vocal sounds outside their innate repertoire (Hayes and Hayes, 1951; Kojima, 2003; Fischer, 2017). This has been ascribed to the lack of an essential anatomical feature of the vocal tract, namely, a decent of the larynx (Lieberman et al., 1969) [see also: (Fitch et al., 2016)], and/or the lack of brain mechanisms responsible for the intricate control of speech organs (Holstege and Subramanian, 2016; Pisanski et al., 2016; Belyk and Brown, 2017). Thus, it is not surprising if their auditory cortical functions are organized differently than in humans, who can produce an infinite number of speech sounds by chaining phonemes in time. Further studies are warranted to elucidate how the elongation of auditory processing time scale supported the human evolution of language and other auditory cognitive abilities.

\section{DATA AVAILABILITY}

The raw data supporting the conclusions of this manuscript will be made available by the authors, without undue reservation, to any qualified researcher.

\section{ETHICS STATEMENT}

This human study was carried out in accordance with the recommendations of the Ethical Guidelines for Medical and Health Research Involving Human Subjects (Ministry of Education, Culture, Sports, Science, and Technology; Ministry of Health, Labor, and Welfare) with written informed consent from all subjects. All subjects gave written informed consent in accordance with the Declaration of Helsinki. The protocol was approved by the Internal Review Board of the University of Niigata. The animal study was carried out in accordance with the recommendations of the Guide for the Care and Use of Laboratory Animals of the National Research Council (1996) and the Guide for Care and Use of Laboratory Primates of Kyoto University. The protocol was approved by the Animal Care and Use Committee of Kyoto University. 


\section{AUTHOR CONTRIBUTIONS}

KI conceived and designed the study. KI, MN, NK, and KN conducted the experiments. KI analyzed the data. KI and TN wrote the draft of the manuscript. All authors contributed to manuscript revision.

\section{REFERENCES}

Alain, C., and McDonald, K. L. (2007). Age-related differences in neuromagnetic brain activity underlying concurrent sound perception. J. Neurosci. 27, 13081314. doi: 10.1523/jneurosci.5433-06.2007

Alain, C., Schuler, B. M., and McDonald, K. L. (2002). Neural activity associated with distinguishing concurrent auditory objects. J. Acoust. Soc. Am. 111, 990995. doi: 10.1121/1.1434942

Alain, C., Woods, D. L., and Covarrubias, D. (1997). Activation of duration-sensitive auditory cortical fields in humans. Electroencephalogr. Clin. Neurophysiol. 104, 531-539. doi: 10.1016/s0168-5597(97) 00057-9

Arezzo, J., Pickoff, A., and Vaughan, H. G. (1975). The sources and intracerebral distribution of auditory evoked potentials in the alert rhesus monkey. Brain Res. 90, 57-73. doi: 10.1016/0006-8993(75)90682-4

Belyk, M., and Brown, S. (2017). The origins of the vocal brain in humans. Neurosci. Biobehav. Rev. 77, 177-193. doi: 10.1016/j.neubiorev.2017. 03.014

Farbood, M. M., Heeger, D. J., Marcus, G., Hasson, U., and Lerner, Y. (2015). The neural processing of hierarchical structure in music and speech at different timescales. Front. Neurosci. 9:157. doi: 10.3389/fnins. 2015.00157

Fastl, H., and Zwicker, E. (2007). Psychoacoustics: Facts and Models. Springer Series in Information Sciences. New York, NY: Springer.

Fischer, J. (2017). Primate vocal production and the riddle of language evolution. Psychon. Bull. Rev. 24, 72-78. doi: 10.3758/s13423-0161076-8

Fishman, Y. I., Reser, D. H., Arezzo, J. C., and Steinschneider, M. (2000a). Complex tone processing in primary auditory cortex of the awake monkey. I. Neural ensemble correlates of roughness. J. Acoust. Soc. Am. 108, 235-246. doi: 10. $1121 / 1.429460$

Fishman, Y. I., Reser, D. H., Arezzo, J. C., and Steinschneider, M. (2000b). Complex tone processing in primary auditory cortex of the awake monkey. II. Pitch versus critical band representation. J. Acoust. Soc. Am. 108, 247-262. doi: $10.1121 / 1.429461$

Fishman, Y. I., and Steinschneider, M. (2012). Searching for the mismatch negativity in primary auditory cortex of the awake monkey: deviance detection or stimulus specific adaptation? J. Neurosci. 32, 15747-15758. doi: 10.1523/ JNEUROSCI.2835-12.2012

Fishman, Y. I., Steinschneider, M., and Micheyl, C. (2014). Neural representation of concurrent harmonic sounds in monkey primary auditory cortex: implications for models of auditory scene analysis. J. Neurosci. 34, 12425-12443. doi: 10. 1523/JNEUROSCI.0025-14.2014

Fitch, W. T., de Boer, B., Mathur, N., and Ghazanfar, A. A. (2016). Monkey vocal tracts are speech-ready. Sci. Adv. 2:e1600723. doi: 10.1126/sciadv. 1600723

Gil-da-Costa, R., Stoner, G. R., Fung, R., and Albright, T. D. (2013). Nonhuman primate model of schizophrenia using a noninvasive EEG method. Proc. Natl. Acad. Sci. U.S.A. 110, 15425-15430. doi: 10.1073/pnas. 1312264110

Gustison, M. L., le Roux, A., and Bergman, T. J. (2012). Derived vocalizations of geladas (Theropithecus gelada) and the evolution of vocal complexity in primates. Philos. Trans. R. Soc. Lond. B Biol. Sci. 367, 1847-1859. doi: 10.1098/ rstb. 2011.0218

Hasson, U., Chen, J., and Honey, C. J. (2015). Hierarchical process memory: memory as an integral component of information processing. Trends Cogn. Sci. 19, 304-313. doi: 10.1016/j.tics.2015.04.006

Hayes, K. J., and Hayes, C. (1951). The intellectual development of a home-raised chimpanzee. Proc. Am. Philos. Soc. 95, 105-109.

\section{FUNDING}

This work was supported by the JSPS KAKENHI (Grant Nos. 25540052, 17K00202, and 19H05309), and Cooperative Research Program of Primate Research Institute, Kyoto University.

Heil, P., and Neubauer, H. (2001). Temporal integration of sound pressure determines thresholds of auditory-nerve fibers. J. Neurosci. 21, 7404-7415. doi: 10.1523/jneurosci.21-18-07404.2001

Hillyard, S. A., and Picton, T. W. (1978). On and off components in the auditory evoked potential. Percept. Psychophys. 24, 391-398. doi: 10.3758/ bf03199736

Holstege, G., and Subramanian, H. H. (2016). Two different motor systems are needed to generate human speech. J. Comp. Neurol. 524, 1558-1577. doi: 10. $1002 /$ cne. 23898

Honing, H., Merchant, H., Haden, G. P., Prado, L., and Bartolo, R. (2012). Rhesus monkeys (Macaca mulatta) detect rhythmic groups in music, but not the beat. PLoS One 7:e51369. doi: 10.1371/journal.pone. 0051369

Itoh, K., Nejime, M., Konoike, N., Nakada, T., and Nakamura, K. (2015). Noninvasive scalp recording of cortical auditory evoked potentials in the alert macaque monkey. Hear. Res. 327, 117-125. doi: 10.1016/j.heares.2015. 05.007

Javitt, D. C., Jayachandra, M., Lindsley, R. W., Specht, C. M., and Schroeder, C. E. (2000). Schizophrenia-like deficits in auditory P1 and N1 refractoriness induced by the psychomimetic agent phencyclidine (PCP). Clin. Neurophysiol. 111, 833-836. doi: 10.1016/s1388-2457(99)00313-2

Kojima, S. (2003). A Search for the Origins of Human Speech: Auditory and Vocal Functions of the Chimpanzee. Kyoto: Academic Press.

Lalitte, P., and Bigand, E. (2006). Music in the moment? Revisiting the effect of large scale structures. Percept. Mot. Skills 103, 811-828. doi: 10.2466/pms.103. 3.811-828

Legatt, A. D., Arezzo, J. C., and Vaughan, H. G. Jr. (1986). Short-latency auditory evoked potentials in the monkey. I. Wave shape and surface topography. Electroencephalogr. Clin. Neurophysiol. 64, 41-52. doi: 10.1016/0013-4694(86) 90042-8

Lerner, Y., Honey, C. J., Katkov, M., and Hasson, U. (2014). Temporal scaling of neural responses to compressed and dilated natural speech. J. Neurophysiol. 111, 2433-2444. doi: 10.1152/jn.00497.2013

Lerner, Y., Honey, C. J., Silbert, L. J., and Hasson, U. (2011). Topographic mapping of a hierarchy of temporal receptive windows using a narrated story. J. Neurosci. 31, 2906-2915. doi: 10.1523/JNEUROSCI.3684-10.2011

Lieberman, P. H., Klatt, D. H., and Wilson, W. H. (1969). Vocal tract limitations on the vowel repertoires of rhesus monkey and other nonhuman primates. Science 164, 1185-1187. doi: 10.1126/science.164.3884.1185

Moore, B. C. J. (2003). Temporal integration and context effects in hearing. J. Phon 31, 563-574. doi: 10.1016/s0095-4470(03)00011-1

Naatanen, R., Paavilainen, P., Rinne, T., and Alho, K. (2007). The mismatch negativity (MMN) in basic research of central auditory processing: a review. Clin. Neurophysiol. 118, 2544-2590. doi: 10.1016/j.clinph.2007. 04.026

Onishi, S., and Davis, H. (1968). Effects of duration and rise time of tone bursts on evoked V potentials. J. Acoust. Soc. Am. 44, 582-591. doi: 10.1121/1. 1911124

Pantev, C., Eulitz, C., Hampson, S., Ross, B., and Roberts, L. E. (1996). The auditory evoked "off" response: sources and comparison with the "on" and the "sustained" responses. Ear Hear. 17, 255-265. doi: 10.1097/00003446199606000-00008

Pisanski, K., Cartei, V., McGettigan, C., Raine, J., and Reby, D. (2016). Voice modulation: a window into the origins of human vocal control? Trends Cogn. Sci. 20, 304-318. doi: 10.1016/j.tics.2016. 01.002

Ross, B., Snyder, J. S., Aalto, M., McDonald, K. L., Dyson, B. J., Schneider, B., et al. (2009). Neural encoding of sound duration persists in older adults. Neuroimage 47, 678-687. doi: 10.1016/j.neuroimage.2009.04.051 
Šidák, Z. (1967). Rectangular confidence regions for the means of multivariate normal distributions. J. Am. Stat. Assoc. 62, 626-633. doi: 10.1080/01621459. 1967.10482935

Sussman, E. S. (2005). Integration and segregation in auditory scene analysis. J. Acoust. Soc. Am. 117, 1285-1298. doi: 10.1121/1.1854312

Teichert, T. (2016). Tonal frequency affects amplitude but not topography of rhesus monkey cranial EEG components. Hear. Res. 336, 29-43. doi: 10.1016/j.heares. 2016.04.001

Woodman, G. F. (2012). "Homologues of Human ERP Components in Nonhuman Primates," in The Oxford Handbook of Event-Related Potential Components, eds S. J. Luck and E. S. Kappenman (Oxford: Oxford University Press), 611-625.
Conflict of Interest Statement: The authors declare that the research was conducted in the absence of any commercial or financial relationships that could be construed as a potential conflict of interest.

Copyright (c) 2019 Itoh, Nejime, Konoike, Nakamura and Nakada. This is an openaccess article distributed under the terms of the Creative Commons Attribution License (CC BY). The use, distribution or reproduction in other forums is permitted, provided the original author(s) and the copyright owner(s) are credited and that the original publication in this journal is cited, in accordance with accepted academic practice. No use, distribution or reproduction is permitted which does not comply with these terms. 